\title{
Electron field emission from SiC nanopillars produced by using nanosphere lithography
}

\author{
Damla Yeşilpınar and Cem Çelebia) \\ Quantum Device Laboratory, Department of Physics, Izmir Institute of Technology, 35430, Urla, Izmir, \\ Turkey
}

(Received 31 March 2017; accepted 12 June 2017; published 28 June 2017)

\begin{abstract}
Field emitter arrays of silicon carbide based nanopillars with high emitter density were fabricated by using a combination of nanosphere lithography and inductively coupled plasma reactive ion etching techniques. The electron field emission characteristics of the produced nanopillars with two different aspect ratios and geometries were investigated, and the obtained results were compared with each other. The authors found that unlike the samples containing low aspect ratio $\mathrm{SiC}$ nanopillars with blunt tip apex, the samples comprising high aspect ratio nanopillars with sharp tip apex generate greater emission currents under lower electric fields. The nanopillars with sharp tip apex produced field emission currents up to $240 \mu \mathrm{A} / \mathrm{cm}^{2}$ under $17.4 \mathrm{~V} / \mu \mathrm{m}$ applied electric field, while the nanopillars with blunt tip apex produced an emission current of $70 \mu \mathrm{A} / \mathrm{cm}^{2}$. The electric fields required to obtain $10 \mu \mathrm{A} / \mathrm{cm}^{2}$ current density are found to be 9.1 and $7.2 \mathrm{~V} / \mu \mathrm{m}$ for the nanopillars with blunt and sharp tip apex, respectively. Time dependent stability measurements yielded stable electron emission without any abrupt change in the respective current levels of both samples. (C) 2017 American Vacuum Society.

[http://dx.doi.org/10.1116/1.4989853]
\end{abstract}

\section{INTRODUCTION}

Field emission electron sources have been extensively studied over the years to be used in numerous applications including a wide range of vacuum micro/nanoelectronics devices such as flat panel displays, ${ }^{1}$ sensors, ${ }^{2-4}$ information storage, ${ }^{5}$ and high-end surface imaging devices, ${ }^{6}$ given their unique and desirable properties such as high coherency and small beam radius of the emitted electrons. The field emission properties of vast number of materials have yet been investigated in numerous forms to achieve stable and high current densities, especially in low electric fields. Its exceptional properties make $\mathrm{SiC}$ a remarkable candidate for being used as electronic device component that is meant to work in harsh environments. ${ }^{7-9}$ Electron field emission properties of both chemically synthesized $\mathrm{SiC}$ nanowires ${ }^{10,11}$ and $\mathrm{SiC}$ micropillars etched on bulk substrates ${ }^{12}$ have been shown to produce high emission currents up to a few amperes per $\mathrm{cm}^{2}$ at moderate electric fields and striking field enhancement factors ranging between $10^{3}$ and $10^{4}$. The main drawback for large field emitter array out of $\mathrm{SiC}$ nanowires is to achieve perpendicularly aligned structures on the cathode electrode with respect to the anode, which drastically limit the field emission current. ${ }^{13}$

Fabrication of such fine, and well organized nanometer scale structures on bulk substrates is possible only by a few methods such as state of the art nanofabrication techniques, including e-beam lithography (EBL) and nanosphere lithography (NSL). NSL has proven itself to be a reliable and fast technique to acquire highly organized nanostructure arrays in large areas with relatively low-cost equipment. With this technique, it is possible to achieve large area

a)Electronic mail: cemcelebi@iyte.edu.tr monolayer self-assembly patterns on the substrate within a course of minutes. The ease of application also makes this technique appealing for many research groups in various areas.

The use of polystyrene (PS) nanospheres have been in the spotlight of many NSL applications, especially as a mask in various forms, given its easily modified shape and diameter. ${ }^{14}$ Although it has proven to be an effective mask for reactive ion etching (RIE) of field emitter arrays of easy-tofabricate materials like $\mathrm{Si}^{15}{ }^{15}$ it is not suitable to use PS nanospheres directly as an etch mask for robust materials like $\mathrm{SiC}$ that require aggressive and highly sophisticated etching processes, which is conventionally masked by nickel (Ni) structures defined only by EBL.

Here, we present an easy to produce and an effective method to print out the nanosphere self-assembly array into an aggressive reactive ion etching compatible $\mathrm{Ni}$ based nanodot hard mask. We assume that with certain modifications, the same method could also be applied to other materials that require different etch masks. We have successfully fabricated two sets of distinct nanopillar structures on $\mathrm{SiC}$ substrates with different aspect ratios and geometries by using this mask. Electron field emission characteristics of the produced $\mathrm{SiC}$ nanopillars were investigated under high vacuum conditions.

\section{EXPERIMENT}

$\mathrm{SiC}$ nanopillars were fabricated using a combination of NSL and inductively coupled plasma (ICP) RIE techniques. In the experiments, we used nominally n-type doped single crystal $6 \mathrm{H}-\mathrm{SiC}$ wafer. As-received wafer was diced into $4 \times 10 \mathrm{~mm}^{2}$ rectangular substrates. Prior to the deposition of PS nanospheres, the substrates were cleaned chemically by solvents in an ultrasonic bath. Following the cleaning 
(a)

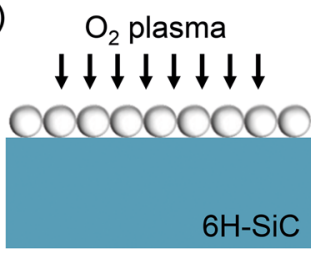

(f)

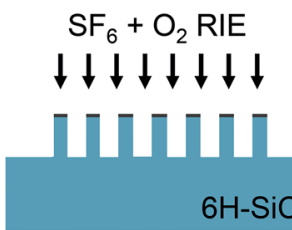

(b)

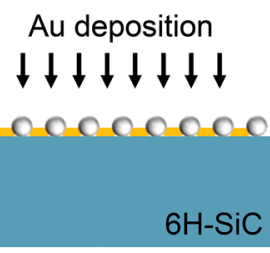

(e)

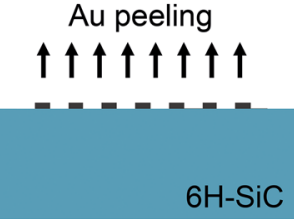

(c)

PS removal

$\uparrow \uparrow \uparrow \uparrow \uparrow \uparrow \uparrow \uparrow$

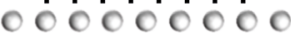

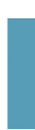

(d)

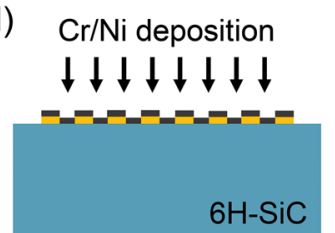

FIG. 1. (Color online) Schematic illustration of the fabrication steps for SiC nanopillar array produced by NSL and ICP-RIE methods. The illustration is not scaled.

procedures, the samples were exposed to $\mathrm{O}_{2}$ plasma in order to convert the hydrophobic character of the SiC surface into hydrophilic. The hydrophilicity of the surface is necessary for binding the hydroxyl groups of PS nanospheres onto the SiC substrate. A solution of nominally $450 \mathrm{~nm}$ diameter PS nanospheres with $10 \mathrm{wt}$. \%/v density in deionized water is diluted by ethanol to get a volume ratio of $7: 1$. The prepared solution of PS nanospheres was spin coated on the surface of $\mathrm{SiC}$ substrate similar to that depicted in Fig. 1(a). The diameter of the nanospheres was reduced by $\mathrm{O}_{2}$ plasma with the parameters of $30 \mathrm{~W}$ power and $8.5 \mathrm{sccm} \mathrm{O}_{2}$ flowrate [Fig. 1(a)]. As the buffer layer which loosely attach on the $\mathrm{SiC}$ surface, a $40 \mathrm{~nm}$ thick $\mathrm{Au}$ thin film was thermally deposited on the samples [Fig. 1(b)]. Then, the samples were immersed in isopropyl alcohol and sonicated for $2 \mathrm{~min}$ to remove the nanospheres with reduced diameters, leaving behind an array of nanoholes on the $\mathrm{SiC}$ substrate [Fig. 1(c)]. Following the nanosphere removal process, $10 / 30 \mathrm{~nm}$ thick $\mathrm{Cr} / \mathrm{Ni}$ film was deposited onto the samples [Fig. 1(d)]. The $\mathrm{Cr} / \mathrm{Ni}$ regions with soft $\mathrm{Au}$ base were peeled out of the SiC surface simply by a sticky tape. Thereby cylindrically shaped approximately $162 \mathrm{~nm}$ diameter $\mathrm{Cr} / \mathrm{Ni}$ based nanodots were obtained [Fig. 1(e)]. Each nanodot serves as a local hard-mask to protect the $\mathrm{SiC}$ regions underneath during the ICP-RIE process. The $\mathrm{SiC}$ substrate with $\mathrm{Cr} / \mathrm{Ni}$ nanodots on its surface was etched by $\mathrm{SF}_{6}+\mathrm{O}_{2}$ gas mixture in a ICP-RIE system. By means of the ICP-RIE process, nanopillars were successfully produced on the $\mathrm{C}$-face surface of the $\mathrm{SiC}$ substrate [Fig. 1(f)]. Every step during the $\mathrm{SiC}$ nanopillar fabrication process was verified by scanning electron microscopy (SEM) measurements. After the SiC based nanopillar fabrication, the samples were immersed into a conventional cheric ammonium nitride/perchloric acid based $\mathrm{Cr}$ etchant solution to remove any remaning traces of the $\mathrm{Cr} / \mathrm{Ni}$ mask.

The SiC substrate with vertically aligned nanopillars on its surface was bonded on a $\mathrm{w}=3 \mathrm{~mm}$ wide planar tungsten plate using a silver paste. As the anode electrode, another tungsten plate was placed at a distance of about $\mathrm{d}=230 \mu \mathrm{m}$ above the sample surface that covers approximately $9 \mathrm{~mm}^{2}$ active emitting area at the middle of the sample. Both electrodes were placed on a home-made vacuum compatible polytetrafluoroethylene based sample holder in order to ensure the electrical isolation between the respective electrodes. The sample stage retaining these two electrodes (Fig. 2) was inserted to a vacuum chamber which can reach a pressure of about $5 \times 10^{-9}$ mbar. The field emission characteristics of the $\mathrm{SiC}$ nanopillars were measured inside the vacuum chamber for an applied potential difference ranging between 0 and $4 \mathrm{kV}$ under the above mentioned pressure range. For the field emission experiments, we used PHYWE 13673-93 high voltage source that is controlled manually, and the field emission currents were read through the voltage drop on a $50 \mathrm{k} \Omega$ load resistance by using KEITHLEY 2182A Nanovoltmeter.

(a)

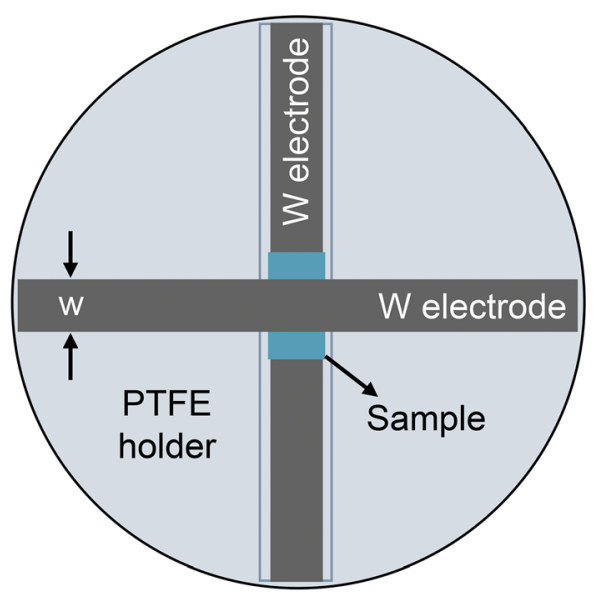

(b)

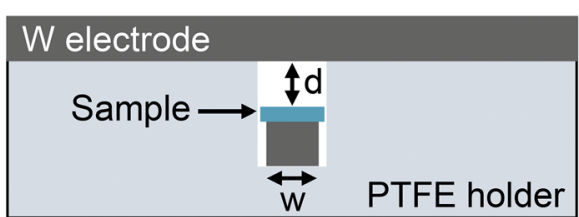

FIG. 2. (Color online) Schematic illustration of the sample stage for electron field emission characterization of $\mathrm{SiC}$ nanopillar array: (a) top view and (b) side view. The illustration is not scaled. 


\section{RESULTS AND DISCUSSION}

Figures 3(a) and 3(b) display the SEM images of spincoated self-assembled monolayer of PS nanospheres on the $\mathrm{SiC}$ surface before and after $\mathrm{O}_{2}$ plasma treatment, respectively. A number of SEM analysis, performed prior to the $\mathrm{O}_{2}$ plasma etching process, revealed an average nanosphere diameter of $453 \mathrm{~nm}$ and a density of $3.9 \times 10^{7} \mathrm{~cm}^{-2}$ on the $\mathrm{SiC}$ surface. The reduction in nanosphere size was measured as a function of the plasma treatment time [Fig. 3(c)], and the obtained data were taken into account for the production of $\mathrm{SiC}$ nanopillars with desired diameter. In our thermal evaporation system, the evaporation direction of the material has a certain angle with respect to the rotating sample holder. Due to the angle and the radius of curvature of the PS nanospheres, for example, with the reduced diameter of $300 \mathrm{~nm}$, the resulting $\mathrm{Cr} / \mathrm{Ni}$ nanodots were found to have an average diameter of about $162 \mathrm{~nm}$ [Figs. 3(b) and 4(a)]. Such a design has allowed us to create even finer structures than defined by the initial diameter of the PS nanospheres, which is indeed desirable for the fabrication of nanoscale structures.

For the fabrication of nanopillars with two distinct geometries and aspect ratios, two sets of samples were etched for the ICP-RIE treatment durations of 1 and $2 \mathrm{~min}$. SEM measurements showed that these two different etching periods yield forests of vertically aligned $\mathrm{SiC}$ nanopillars with low (for $1 \mathrm{~min}$ ) and high (for $2 \mathrm{~min}$ ) aspect ratios (height to
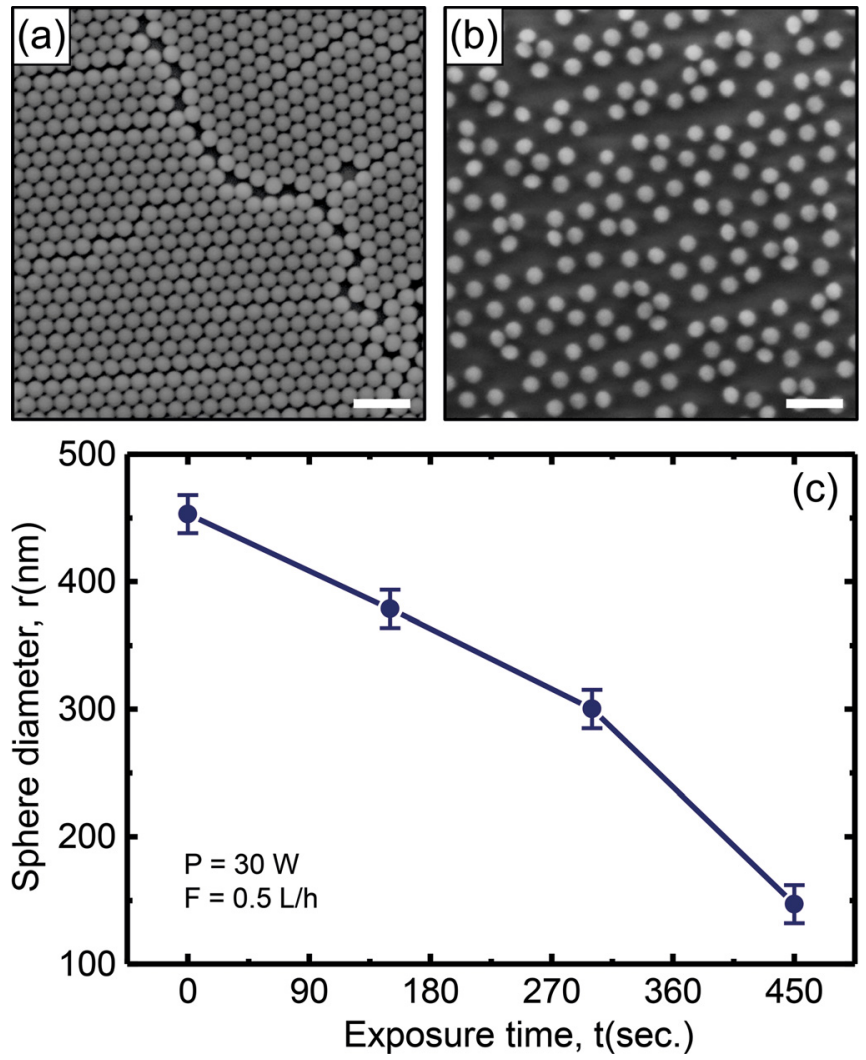

FIG. 3. (Color online) SEM images of (a) self-assembled monolayer pattern of $450 \mathrm{~nm}$ PS nanospheres on $\mathrm{SiC}$ acquired by spin coating (scale bar $1.5 \mu \mathrm{m}$ ) (b) PS nanospheres after $300 \mathrm{~s} \mathrm{O}_{2}$ plasma treatment. Nanosphere diameter reduced to $300 \mathrm{~nm}$ (scale bar $1 \mu \mathrm{m}$ ). (c) The change in the PS nanosphere diameter as a function of $\mathrm{O}_{2}$ plasma treatment time.
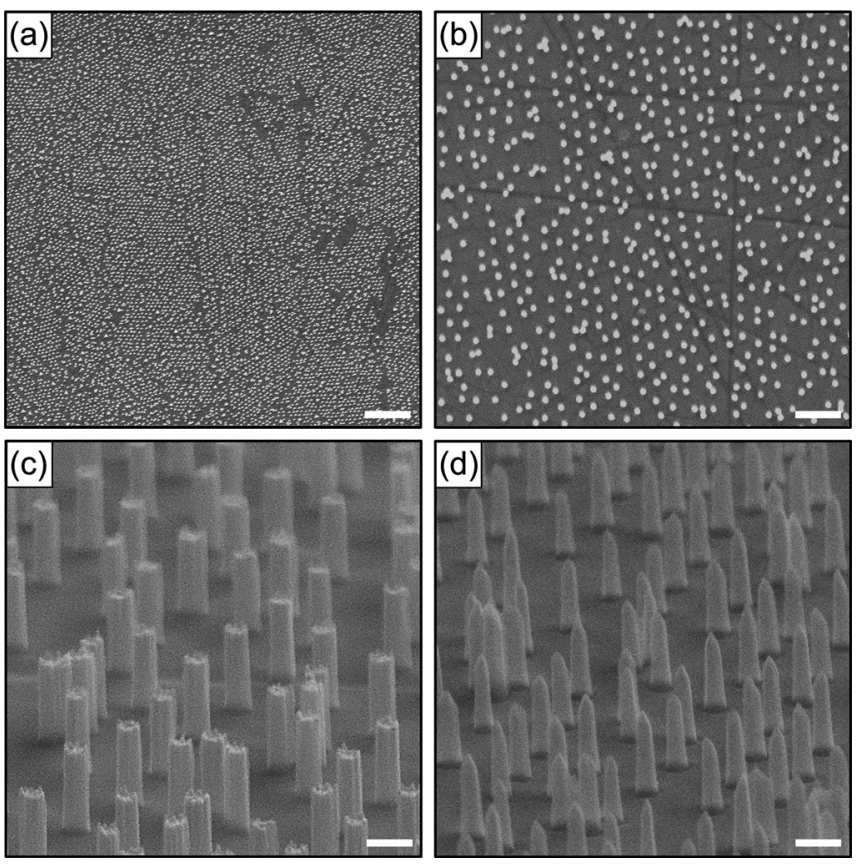

FIG. 4. SEM images (a) and (b) of $\mathrm{Cr} / \mathrm{Ni}$ etch mask on $\mathrm{SiC}$ [scalebars (a) $5 \mu \mathrm{m}$ (b) $1.2 \mu \mathrm{m}$ ] (c) of low aspect ratio nanopillars (NPL) after $1 \mathrm{~min}$ ICPRIE (scalebar $240 \mathrm{~nm}$, angle $70^{\circ}$ ) (d) of high aspect ratio nanopillars (NPH) after 2 min ICP-RIE (scalebar $240 \mathrm{~nm}$, angle $70^{\circ}$ ).

width ratios). Depending on the nanopillar aspect ratios and geometries, the samples were denoted as nanopillar low (NPL) and nanopillar high (NPH). The NPL samples contain low aspect ratio $(\sim 3.3)$ nanopillars with blunt tip apex whereas NPH samples comprise high aspect ratio $(\sim 4.9)$ nanopillars with sharp tip apex with an estimated apex radius of $18 \pm 4 \mathrm{~nm}$, as displayed in Figs. 4(c) and 4(d), respectively. The sharp end geometry of the nanopillars on NPH samples suggests that the shadow mask was completely etched away during the RIE process, resulting in a shorter pillar and a lower aspect ratio than initially expected for 2 min etching time.

As shown in Fig. 5, the field emission characteristics of both NPL and NPH samples were measured as a function of

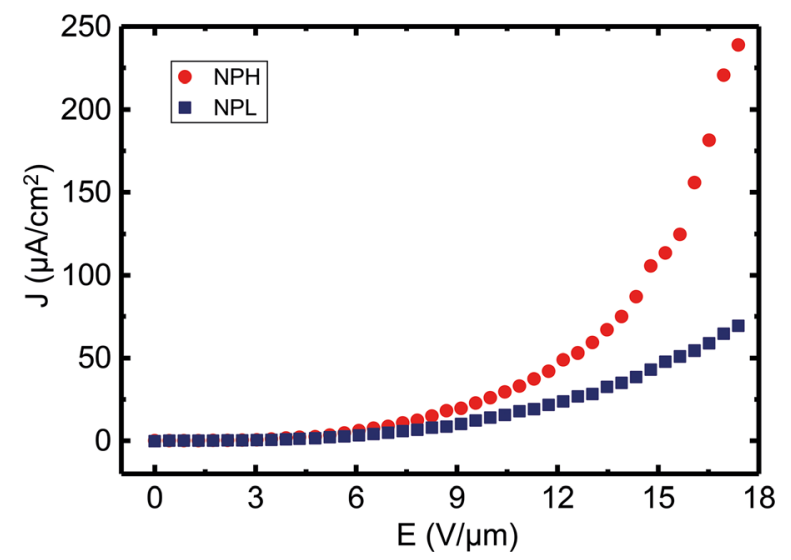

FIG. 5. (Color online) Change in field emission current density with respect to applied electric field for NPL and NPH samples. NPH sample has lower turn-on electric field $(3.4 \mathrm{~V} / \mu \mathrm{m})$ compared to NPL sample $(4.2 \mathrm{~V} / \mu \mathrm{m})$. (The data show a representative sweep-up measurement for each sample.) 
the applied electric field for a constant electrode spacing of $230 \mu \mathrm{m}$ from an active emitting area of about $9 \mathrm{~mm}^{2}$ which is limited by the width (w) of the tungsten electrodes. The turn-on electric fields, defined as the electric field required to obtain an electric current density of $1 \mu \mathrm{A} / \mathrm{cm}^{2}$, are found to be 4.2 and $3.4 \mathrm{~V} / \mu \mathrm{m}$ for the NPL and NPH samples, respectively. In addition, for the NPH sample, the electric field required to reach a current density of $10 \mu \mathrm{A} / \mathrm{cm}^{2}$ is determined to be $7.2 \mathrm{~V} / \mu \mathrm{m}$ which is smaller than the one measured for the NPL sample $(9.1 \mathrm{~V} / \mu \mathrm{m})$ due to its sharp geometry and smaller radius of curvature at the tip apex. The measured total current for NPL is $6.3 \mu \mathrm{A}$ and for NPH is $21.6 \mu \mathrm{A}$. Considering the active emitting area of the samples, the current densities are determined. At the maximum applied electric field of $17.4 \mathrm{~V} / \mu \mathrm{m}$, which is limited by our voltage-current measurement set-up, the field emission current densities were found as 70 and $240 \mu \mathrm{A} / \mathrm{cm}^{2}$ for the NPL and NPH samples, respectively.

The emission current due to the tunneling of electrons under an applied electric field follows the Fowler-Nordheim (FN) equation given as

$$
\mathrm{J}=\frac{\mathrm{A} \beta^{2} \mathrm{E}^{2}}{\phi} \exp \left(-\frac{\mathrm{B} \phi^{3 / 2}}{\beta \mathrm{E}}\right),
$$

where $\mathrm{J}$ is the current density, $\phi$ is the work function of the emitter material, $\mathrm{E}$ is the applied electric field, $\mathrm{A}=1.546$ $\times 10^{-6} \mathrm{~A} \mathrm{~V}^{-2} \mathrm{eV}$, and $\mathrm{B}=6.83 \times 10^{7} \mathrm{eV}^{-3 / 2} \mathrm{~V} \mathrm{~cm}^{-1}$ are the FN constants ${ }^{16}$ and $\beta$ is the field enhancement factor. For a metallic single emitter, $\beta$ is in agreement with the height to apex radius ratio of the emitter. ${ }^{17-19}$ However, for large area field emitter arrays, experimentally acquired values of the $\beta$ factors are observed to have a twofold hierarchy: ${ }^{12}$ (1) the local field enhancement that arise from the individual shape of the emitter structures ${ }^{20}$ and (2) the global electric field enhancement which is related to the spatial arrangement of the field emitters on the sample surface. ${ }^{20-22}$ In the case of semiconductor based field emitters, $\beta$ values calculated from the slopes of the FN plot is observed to deviate from the actual field enhancement of the structure due to the field penetration effect, presence of shallow states arising from the doping of the semiconductor material ${ }^{13,23,24}$ and the crystallinity ${ }^{25}$ of the sample. The combination of these effects may give rise to drastic variations in the experimentally obtained $\beta$ values for large area semiconductor based field emitter arrays. It should be noted that, in this work, we have investigated only the effect of aspect ratio and the geometry of the individual nanoscale emitters in a large area electron field emitter array.

According to the FN theory, the plot of $\ln \left(\mathrm{J} / \mathrm{E}^{2}\right)$ versus $1 / \mathrm{E}$ should give a straight line with a slope of $\mathrm{S}=-\mathrm{B} \phi^{3 / 2} / \beta$. The linear behavior of the $\ln \left(\mathrm{J} / \mathrm{E}^{2}\right)$ to $1 / \mathrm{E}$ plot for our samples shows a good agreement with the FN tunneling phenomenon at the tip apex of the $\mathrm{SiC}$ nanopillar emitters (Fig. 6). Two distinct slopes were observed with a switch at the electric field values between 8.7 and $9.1 \mathrm{~V} / \mu \mathrm{m}$ for both samples (inset of Fig. 6). This steplike behavior was attributed to

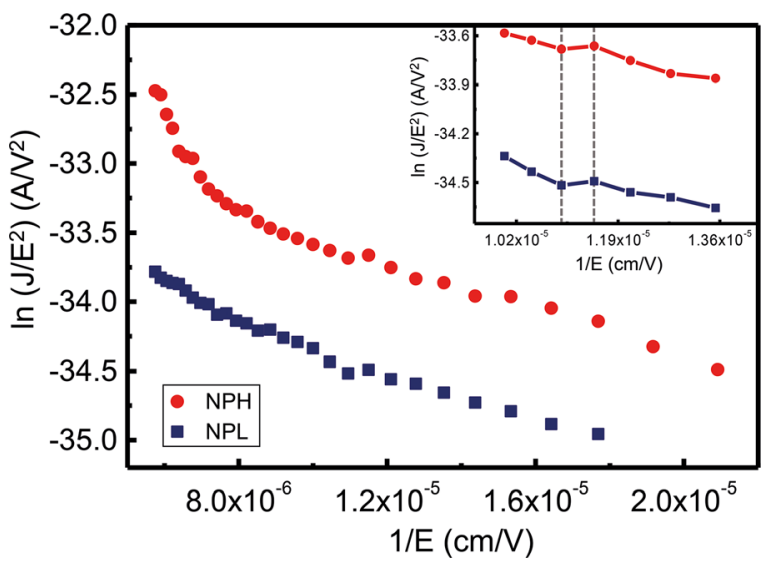

FIG. 6. (Color online) Fowler-Nordheim characteristics of the NPL and NPH samples. (Inset) Step like change observed between the electric field values 8.7 and $9.1 \mathrm{~V} / \mu \mathrm{m}$.

the sudden change in the work function of the samples at relatively high electric fields. ${ }^{25,26}$ The emitted electrons at low fields are more likely to be arising from the surface states where the work function is inherently lower, whereas the main contribution to the field emission current at higher fields is supplied by the bulk. The $\beta$ values were denoted as $\beta^{\mathrm{LE}}$ and $\beta^{\mathrm{HE}}$ for low electric field and high electric field regions, respectively. For the NPL samples, the $\beta$ values were determined as $\beta_{\mathrm{NPL}}^{\mathrm{LE}}=7828$ and $\beta_{\mathrm{NPL}}^{\mathrm{HE}}=4466$ and for the NPH samples as $\beta_{\mathrm{NPH}}^{\mathrm{LE}}=7251$ and $\beta_{\mathrm{NPH}}^{\mathrm{HE}}=5639$, for $\phi=4.2 \mathrm{eV}$ at the C-terminated face of $6 \mathrm{H}-\mathrm{SiC}$ wafer. ${ }^{12}$ For the low electric field values, the $\beta$ parameters have similar results given to presence of surface states, and the aspect ratio does not seem to have an effect on the field emission current due to over-prediction of the experimentally obtained $\beta$ values, whereas at high electric fields, both the aspect ratio and the geometry seem to play an important role. It is clear from the calculated values that the experimentally obtained $\beta$ factors for low electric fields may lead to misinterpretation of the field enhancement phenomena at the emitter tips. It is known that at low electric field values, unintentional contributions from the surface states of the two samples, which were produced for two different ICP-RIE durations, may give rise to such deviations in the calculated field enhancement factors. ${ }^{27}$ Such large field enhancement factor for $\mathrm{n}$ type $6 \mathrm{H}-\mathrm{SiC}$ micropillar structure was previously reported. ${ }^{12}$ However, the field enhancement factors obtained in our study are much higher. It is most likely that the nanoscale roughness ${ }^{18}$ on the nanopillars for the NPL sample, and sharp tip apex with an average radius low as $18 \mathrm{~nm}$ (Refs. 25 and 28) for the NPH sample have acted as effective emission sites which yield higher field enhancement than initially expected from the geometry of the resulting structures. It should be noted that the field enhancement values for NPL $\left(\beta_{\mathrm{NPL}}^{\mathrm{HE}}=4466\right)$ and $\mathrm{NPH}\left(\beta_{\mathrm{NPH}}^{\mathrm{HE}}=5639\right)$ do not correspond to the theoretically obtained field enhancement factors at the emitter tips, and thus further investigations are required to better understand the possible mechanisms behind this peculiar phenomenon. Our measurements explicitly showed that the field enhancement can be further boosted with denser 


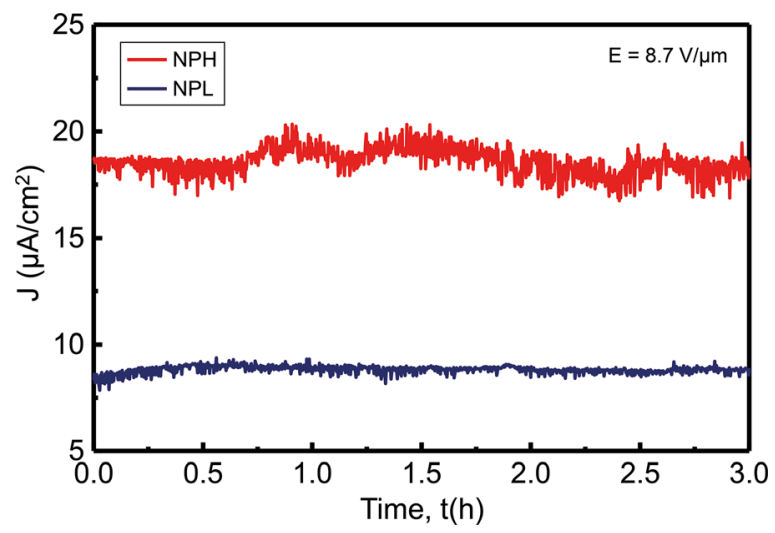

FIG. 7. (Color online) Time dependent measurements of the field emission current stability for NPL $( \pm 8.1 \%)$ and for NPH $( \pm 8.3 \%)$ aquired under an applied electric field of $8.7 \mathrm{~V} / \mu \mathrm{m}$.

and finer structured SiC pillars with adequate spacing to avoid the screening effects. As seen in Fig. 6, the FN plot of the NPH sample shows a deviation from the linearity at high electric fields above $15 \mathrm{~V} / \mu \mathrm{m}$. This behavior is attributed to the joule heating of the sample and thus to the generation of hot electrons. This effect was also observed as a slight increase in the pressure level of our vacuum system.

The stability measurements (Fig. 7) that were conducted for a duration of $3 \mathrm{~h}$ under $8.7 \mathrm{~V} / \mu \mathrm{m}$ electric field revealed reasonably stable electron emission current densities of $8.8 \mu \mathrm{A} / \mathrm{cm}^{2}$ for NPL and $18.5 \mu \mathrm{A} / \mathrm{cm}^{2}$ for NPH, with fluctuations that are calculated from the minimum and maximum currents recorded during the measurements, in the range of $\pm 8.1 \%$ and $\pm 8.3 \%$, respectively. The control measurements taken after $3 \mathrm{~h}$ revealed a similarly stable current levels with congruent fluctuations. No abrupt changes or cut-off in the emission currents were observed for both samples.

\section{CONCLUSION}

We have demonstrated a fast and simple method to produce $\mathrm{Cr} / \mathrm{Ni}$ based nanoscale shadow mask patterns that are capable of resisting to aggressive dry etching processes. A dense array of $\mathrm{SiC}$ based nanopillars, with two different aspect ratios (3.3 and 4.9) and distinct geometries (blunt and sharp tip apex), was fabricated on the C-terminated face of two sets of $6 \mathrm{H}-\mathrm{SiC}$ substrates by using a combination of NSL and ICP-RIE techniques. The field emission characteristics of the produced samples were measured under the influence of the electric fields generated in a voltage range between 0 and $4 \mathrm{kV}$. The turn-on electric field and the electric field required to obtain a current density of $10 \mu \mathrm{A} / \mathrm{cm}^{2}$ for low aspect ratio samples with blunt tip apex (NPL) were found to be 4.2 and $9.1 \mathrm{~V} / \mu \mathrm{m}$, respectively. These values are observed to be lowered down to 3.4 and $7.2 \mathrm{~V} / \mu \mathrm{m}$ for the high aspect ratio samples with sharp tip apex (NPH). Both samples yielded stable current densities over a $3 \mathrm{~h}$ of period. Further stability measurements carried out for longer time of periods did not reveal any cut-offs or abrupt changes in the $\mathrm{SiC}$ nanopillar emission currents.

\section{ACKNOWLEDGMENTS}

The authors would like to thank Cenk Yanık from Sabanc1 University Nanotechnology Research and Application Center (SUNUM) and Sirrı Batuhan Kalkan from İzmir Institute of Technology (IZTECH) for assistance and help in the sample preparation procedures. SEM analyses of the samples were done in the Center for Materials Research of Izmir Institute of Technology. This work was financially supported by the Scientific and Technological Research Council of Turkey under project Grant No. TÜBITAK-115F092.

${ }^{1}$ S. Uemura, J. Yotani, T. Nagasako, H. Kurachi, H. Yamada, T. Ezaki, T. Maesoba, and T. Nakao, Dig. Tech. Pap.-Soc. Inf. Disp. Int. Symp. 33, 1132 (2002).

${ }^{2}$ C. Langer, C. Prommesberger, and R. Schreiner, in 2016 29th International Vacuum Nanoelectronics Conference (IEEE, Vancouver, BC, 2016), pp. 6-7.

${ }^{3}$ S. Wilfert and C. Edelmann, Vacuum 86, 556 (2012).

${ }^{4}$ W. Knapp, D. Schleussner, and M. Wüest, J. Phys. Conf. Ser. 100, 092007 (2008).

${ }^{5}$ S. Lam, 14th International Vac. Microelectron. Conf. (IEEE, Davis, CA, 2001), pp. 135-136.

${ }^{6}$ H. Nakahara, S. Ichikawa, T. Ochiai, Y. Kusano, and Y. Saito, e-J. Surf. Sci. Nanotechnol. 9, 400 (2011).

${ }^{7}$ M. Mehregany, C. A. Zorman, N. Rajan, and C. H. Wu, Proc. IEEE 86, 1594 (1998).

${ }^{8}$ R. G. Azevedo et al., IEEE Sens. J. 7, 568 (2007).

${ }^{9}$ D. G. Senesky, B. Jamshidi, and A. P. Pisano, IEEE Sens. J. 9, 1472 (2009).

${ }^{10}$ G. Shen, Y. Bando, C. Ye, B. Liu, and D. Golberg, Nanotechnology 17, 3468 (2006)

${ }^{11}$ W. M. Zhou, Y. J. Wu, E. S. W. Kong, F. Zhu, Z. Y. Hou, and Y. F. Zhang, Appl. Surf. Sci. 253, 2056 (2006).

${ }^{12}$ M. G. Kang, H. Lezec, R. L. Kallaher, and F. Sharifi, Proc. IEEE Conf. Nanotechnol. 65201, 2 (2012).

${ }^{13}$ X. Zhang, Y. Chen, Z. Xie, and W. Yang, J. Phys. Chem. C 114, 8251 (2010).

${ }^{14}$ S. S. Shinde and S. Park, J. Semicond. 36, 23001 (2015).

${ }^{15}$ W. Li et al., Nanotechnology 19, 135308 (2008).

${ }^{16}$ D. Mihalcea and P. Piot, 14th Conference On Linear Accelerator (TRIUMF, Victoria, BC, 2008), pp. 652-654.

${ }^{17}$ S. Podenok, M. Sveningsson, K. Hansen, and E. E. B. Campbell, Nano 1, 87 (2006).

${ }^{18}$ R. G. Forbes, C. Edgcombe, and U. Valdrè, Ultramicroscopy 95, 57 (2003).

${ }^{19}$ C. Langer, C. Prommesberger, F. Dams, and R. Schreiner, i25th International Vacuum Nanoelectronics Conference (IEEE, Jeju, 2012), pp. $1-2$.

${ }^{20}$ J.-M. Bonard, N. Weiss, H. Kind, T. Stöckli, L. Forró, K. Kern, and A. Châtelain, Adv. Mater. 13, 184 (2001).

${ }^{21}$ X. Wang, J. Zhou, C. Lao, J. Song, N. Xu, and Z. L. Wang, Adv. Mater. 19, 1627 (2007).

${ }^{22}$ J. O. Hwang, D. H. Lee, J. Y. Kim, T. H. Han, B. H. Kim, M. Park, K. No, and S. O. Kim, J. Mater. Chem. 21, 3432 (2011).

${ }^{23}$ V. A. Gubanov and C. Y. Fong, Appl. Phys. Lett. 75, 88 (1999).

${ }^{24}$ S. Chen, M. Shang, Z. Yang, J. Zheng, L. Wang, Q. Liu, F. Gao, and W. Yang, J. Mater. Chem. C 4, 7391 (2016).

${ }^{25}$ H.-Y. Hsieh, S.-H. Huang, K.-F. Liao, S.-K. Su, C.-H. Lai, and L.-J. Chen, Nanotechnology 18, 505305 (2007).

${ }^{26}$ W. Wang and Z. Li, OALib, arXiv:1211.4197, 6 (2012).

${ }^{27}$ G. S. Oehrlein, Mater. Sci. Eng. B 4, 441 (1989).

${ }^{28}$ Y. M. Chang, M. C. Liu, P. H. Kao, C. M. Lin, H. Y. Lee, and J. Y. Juang, ACS Appl. Mater. Interfaces 4, 1411 (2012). 